\title{
Triple-Rule-Out Computed Tomography Scanning for the Diagnosis of a Mediastinal Tumor
}

\author{
Dritan Useini $^{1} \quad$ Zulfugar Taghiyev $^{1}$ Matthias Bechtel ${ }^{1} \quad$ Justus Strauch $^{1}$ \\ 1 Department of Cardiothoracic Surgery, Ruhr-University Hospital \\ Bergmannsheil, Bochum, Germany \\ Thorac Cardiovasc Surg Rep 2019;8:e30-e32. \\ Address for correspondence Dritan Useini, MD, Department of \\ Cardiothoracic Surgery, Ruhr-University Hospital Bergmannsheil, \\ Bürkle-de-la-Camp-Platz-1, Bochum 44789, Germany \\ (e-mail: dritan-83@live.com).
}

\begin{abstract}
Keywords

- thymus

- computed tomography

- TRO-CT scan

Thymomas are rare tumors that commonly lie in the anterior mediastinum. The arteries supplying thymomas branch from the internal thoracic arteries and their collaterals. This report presents a patient with huge mediastinal thymoma, whose sensational vascularization is ensured directly by a single dominant tributary branch of the left internal thoracic artery. After initially performed computed tomography angiography, the tumor was suspect to be vascularized from the right coronary artery. Triple-rule-out computed tomography scan was a key step for accurate radiologic diagnosis.
\end{abstract}

\section{Introduction}

The thymomas can grow in giant dimensions, while current computed tomography angiography (CTA) is the imaging method of choice for evaluating thymoma and preoperative preparation. ${ }^{1}$ We present the value of triple-rule-out computed tomography (TRO-CT) scan for further diagnosis of mediastinal thymoma, when the standard CTA fails to provide details, important for cardiothoracic interventions.

\section{Case Report}

A 63-year-old woman was referred to our hospital after an abnormal chest roentgenogram finding during an acute bronchitis. For further evaluation, the chest roentgenogram was followed by CTA in a peripheral radiological institute. A mediastinal tumor was found. Due to suspected arterial vascularization of the tumor from the right coronary artery ( - Fig. 1A) and as a surgical resection was indicated, we needed to distinguish the arterial blood supply of the tumor. For further investigation, TRO-CT scan was employed. The TRO-CT scan revealed anatomical details relating to tumor invasiveness and relationships to other surrounding structures with respect to the heart, coronaries, pulmonary arteries, and great blood vessels. The surprising and unique finding was the tumor's vascularization. We saw how a single big artery branching from the left internal thoracic artery (LITA) was removed through the right parts of the anterior mediastinum, vascularizing the tumor directly $(\mathbf{- F i g . 1 B / C})$. As we excluded a coronary vascularization of the tumor and infiltration of the tumor to the heart and great blood vessels (-Fig. 1D), the surgical access to the tumor was accomplished through the upper partial J-sternotomy. Due to tumor noninvasiveness, the extirpation was unspectacular. The histopathological analysis of the extirpated mass, measuring $11 \times 9.5 \times 6.5 \mathrm{~cm}$ ( - Fig. 2 B), revealed AB thymoma according to the World Health Organization classification and Masaoka-Koga stage I (-Fig. 2A).

\section{Discussion}

The importance of TRO-CT in the emergency departments is uncontroversial. TRO-CT is a tailored electrocardiography (ECG)-gated examination designed to evaluate the aorta, coronary circulation, and pulmonary arteries using either a high volume or a double injection of contrast media. For the patients with unclear chest pain, coronary artery disease, pulmonary embolization, and acute aortic dissection can be ruled out. CTA represents the first line choice for evaluating a mediastinal received

May 28, 2019

accepted after revision

July 30, 2019
DOI https://doi.org/

10.1055/s-0039-1697602. ISSN 2194-7635. (c) 2019 Georg Thieme Verlag KG
Stuttgart · New York

License terms

c) $(1) \$ \$$ 

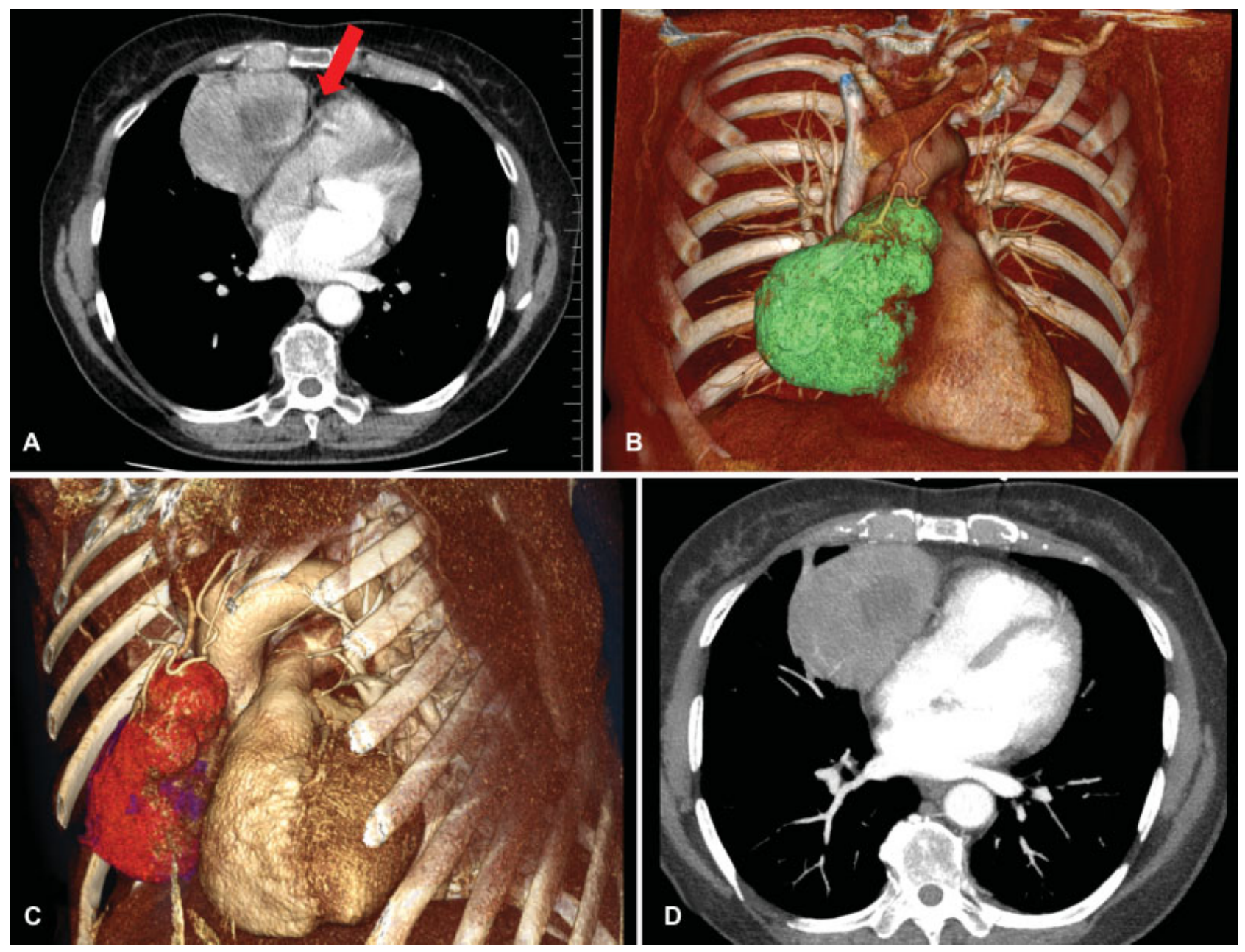

Fig. 1 (A) Computed tomography (CT) angiography shows the tumor mass in an anatomical relationship with the right heart. Here, a tumor vascularization from the right coronary artery was presumed (red arrow). (B/C) Triple-rule-out-computed tomography (TRO-CT) threedimensional reconstruction showing a big tributary branch of left internal thoracic artery, displaced in the right parts of the anterior mediastinum, directly penetrating and vascularizing the tumor mass, and how the tumor vein opens into the brachiocephalic vein. (D) TRO-CT scan excluding any infiltration of the heart and great blood vessels and vascularization of the tumor from coronary artery.

tumor mass. ${ }^{1}$ However, a recent study indicated that even after examining with contrast-enhanced CTA, the incidence of incomplete tumor resectability of mediastinal thymoma remains quite high (17.3\%). In 133 patients diagnosed with thymomas, the abutment of the circumference of an adjacent vessel, revealed in contrast-enhanced CTA, contributed significantly to incomplete surgical resection. ${ }^{2}$ Utilizing TRO-CT might improve the operative strategy to increase the surgical success in tumors close to the heart and the great vessels because of the tailored ECG guidance and, consequently, better resolution.

In general, for radiological evaluation of the mediastinal tumors, standard CTA is used with varying degrees of contrast

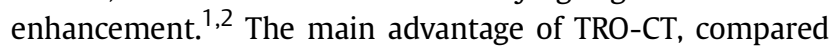
with standard/contrast-enhanced CTA, is the tailored ECG guidance, which provides high resolution of the heart and the great vessels and, as a result stricter differentiation can be made between the tumors that lie close to the heart and heart itself, with respect to the tumor invasiveness. Second, TRO-CT has clearly defined volume and way of application of the contrast medium to expose all heart chambers and great vessels, contrary to standard CTA where due to the dependence on contrast enhancement the right heart chambers might not be seen. This might have implications on defining vasculature relationships of the tumors with the heart and the great vessels. Third, with TRO-CT exposing the whole thoracic aortic stem, all arterial branches (originating from arteria thoracica interna, truncus brachiocephalicus, etc.) supplying blood to the tumor can be visualized.

Tumors where arterial blood supply originates from the coronary arteries are very rare. Interestingly, even in the last year's published cases ${ }^{3-5}$ the CTA as a radiological tool failed to provide imaging of coronary blood supply to the tumor. In all cases, a left heart catheterization for direct coronarography was necessary for further diagnostics. We examined TRO-CT to distinguish whether the blood supply of the tumor mass originated from the coronary artery. This accurate method excluded tumorous vascularization from coronary artery. On the other hand, it identified very clearly the relationships of the mass with adjacent structures, including the large mediastinal blood vessels and right ventricle. Moreover, a coronary artery disease was excluded and a pathoanatomic insight was discovered. 

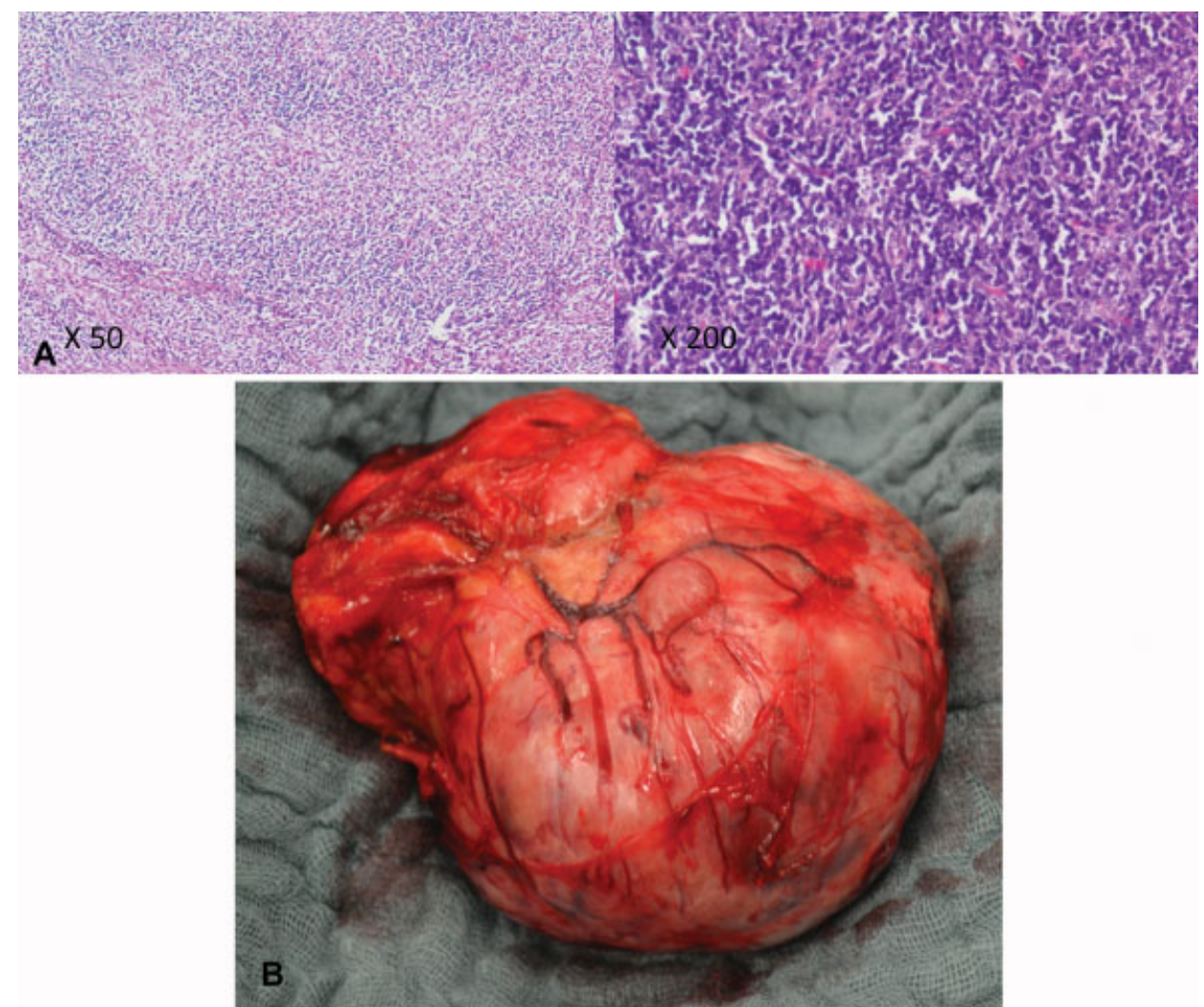

Fig. 2 (A) Hematoxylin and eosin stain of type AB thymoma with areas consists of whorls of spindle thymic epithelial cells surrounded by areas of lymphoid stroma; magnification $\times 50 / x$ 200. (B) Extirpated tumor, measuring $11 \times 9.5 \times 6.5 \mathrm{~cm}$.

The vascularization after tumorous alteration and enlargement of the thymus gland is very sparsely described. The modest literature concerning this points out that the feeding vessels of the thymomas branch from LITA and collateral blood vessels. ${ }^{6,7}$ In another report, it has been discovered that the vascularization of a thymoma originates from an aortocoronaric bypass graft. ${ }^{8}$ In our case, we found a surprising and unique vascularization of the thymoma. A big tributary branch of LITA, comparable to LITA, was displaced in the right parts of the anterior mediastinum, directly penetrating and vascularizing the tumor mass.

\section{Conclusion}

In cases where standard/contrast-enhanced CTA fails to provide sufficient imaging, TRO-CT scan should be used in designing a cardiothoracic operative strategy for mediastinal tumors with anatomical relationships very close to the heart and the great vessels. It may even be helpful in correct anatomical and embryological evaluations of mediastinal masses. Furthermore, attendant diseases, such as coronary artery disease, could also be detected.

Conflict of Interest

None.

\section{References}

1 Benveniste MF, Rosado-de-Christenson ML, Sabloff BS, Moran CA, Swisher SG, Marom EM. Role of imaging in the diagnosis, staging, and treatment of thymoma. Radiographics 2011;31(07):1847-1861, discussion 1861-1863

2 Hayes SA, Huang J, Plodkowski AJ, et al. Preoperative computed tomography findings predict surgical resectability of thymoma. J Thorac Oncol 2014;9(07):1023-1030

3 González-Santos JM, Arnáiz-García ME, Muñoz-Herrera Á, López-Rodríguez J. Mediastinal paraganglioma fed by the left circumflex artery. Interact Cardiovasc Thorac Surg 2016;23(05): 835-836

4 Ho MY, Fleischmann D, Forrester MD, Lee DP. Coil embolization of a left circumflex feeder branch in a patient with a mediastinal paraganglioma. JACC Cardiovasc Interv 2011;4(12): 1345-1346

5 Qedra N, Kadry M, Ivanitskaia-Kühn E, et al. Solitary fibrous mediastinal tumor with coronary vascular supply: an unusual case. J Thorac Cardiovasc Surg 2010;139(02):e23-e25

6 Takenaka T, Ishida T, Handa Y, Tsutsui S, Matsuda H. Ectopic thymoma presenting as a giant intrathoracic mass: a case report. J Cardiothorac Surg 2012;7:68

7 Andreini D, Pontone G, Dainese L, et al. Preoperative assessment of thymoma: evaluation of mediastinal arterial anatomy by cardiac multidetector computed tomography. J Thorac Imaging 2009;24(01):31-33

8 Thomas PA, Collart F, Doddoli C, Gariboldi V, Moulin G. Nourishing vascularization of a thymoma issued from a left internal thoracic artery graft. J Thorac Cardiovasc Surg 2006;131(05):1196-1197 